\title{
Depressive symptoms but not chronic pain have an impact on the survival of patients undergoing maintenance hemodialysis
}

\author{
Mariusz Kusztal ${ }^{1}$, Ewa Trafidło ${ }^{2}$, Katarzyna Madziarska ${ }^{1}$, Hanna Augustyniak-Bartosik' \\ Maciej Karczewski ${ }^{3}$, Waclaw Weyde ${ }^{4}$, Magdalena Krajewska ${ }^{1}$, Joanna Rymaszewska ${ }^{5}$, Marian Klinger $^{1}$
}

\author{
${ }^{1}$ Department of Nephrology and Transplantation Medicine, Wroclaw Medical \\ University, Wroclaw, Poland \\ ${ }^{2} \mathrm{FMC}$ Poland, Swidnica, Poland \\ ${ }^{3}$ Manteion - Statistical Laboratory, Regional Specialist Hospital in Wroclaw, Research \\ and Development Center, Poland \\ ${ }^{4}$ Faculty of Dentistry, Wroclaw Medical University, Wroclaw, Poland \\ ${ }^{5}$ Department of Psychiatry, Wroclaw Medical University, Wroclaw, Poland
}

Submitted: 2 August 2015

Accepted: 5 October 2015

Arch Med Sci 2018; 14, 2: 265-275

DOI: 10.5114 /aoms.2016.59765

Copyright (c) 2016 Termedia \& Banach

\begin{abstract}
Introduction: More than 1/3 of patients with end-stage renal disease who are in a chronic dialysis program suffer from chronic pain and depression/anxiety. The aim of the study was to determine the impacts of symptoms of depression/anxiety, chronic pain and quality of life (QoL) on 6-year patient survival. Material and methods: Observational study of end-stage renal disease patients on maintenance hemodialysis $(n=205)$ who met the inclusion criteria. Patients from three dialysis centers in Lower Silesia were asked to complete a battery of validated questionnaires: the Hospital Anxiety and Depression Scale (HADS), the 36-item Short Form Health Survey Questionnaire, the Verbal Rating Scale (VRS) and the Visual Analog Scale (VAS). Clinical and biochemical data (dialysis adequacy) were recorded.

Results: One hundred thirty from 205 enrolled hemodialysis patients (63.4\%) suffered from chronic pain. Patients with pain were on maintenance dialysis for longer times and had higher levels of parathyroid hormone, more depressive symptoms and a lower QoL than those without pain. In the 6-year period, $96(46.8 \%)$ patients died. The most common cause of death was cardiovascular disease in $44(45.8 \%)$ patients. Highly depressed patients (HADS depression score $>8$ ) exhibited higher mortality ( $<8$ vs. $>8$ points; $p=0.016)$ independent of age, diabetes, cardiovascular disease, C-reactive protein or albumin level.

Conclusions: Chronic pain, although common among hemodialysis patients, did not lower survival. Depressive symptoms are an important predictor for all-cause mortality in hemodialysis patients, with the relationship independent of nutritional or inflammatory status.
\end{abstract}

Key words: chronic pain, depressive symptoms, hemodialysis, quality of life, mortality.

\section{Introduction}

All-cause mortality among dialysis patients remains high despite improvements in technology and management of anemia and disturbances in calcium-phosphate metabolism. This is partly due to the increasing

\author{
Corresponding author: \\ Mariusz Kusztal MD, PhD \\ Department of Nephrology \\ and Transplantation Medicine \\ Wroclaw Medical University \\ 213 Borowska St \\ 50-556 Wroclaw, Poland \\ Phone: +48 71609052969 \\ +48717332515 \\ Fax: +48 717332509 \\ E-mail: mariok@tlen.pl
}


number of elderly patients with many comorbidities enrolled in dialysis programs [1]. The overall symptom burden of these patients is high and is similar to that of the end-of-life cancer population.

Determinants of mortality in patients with end-stage renal disease (ESRD) treated using hemodialysis include older age, nutritional status and comorbidities such as diabetes mellitus and inflammation [2, 3]. Psychosocial factors, such as depression $[4,5]$ and quality of life (QoL) covering self-reported bodily pain [6, 7], have also been associated with mortality in dialysis patients.

The prevalence of depression verified by a specialist in patients undergoing hemodialysis ranges from $5 \%$ to $30 \%$, but depressive/anxiety symptoms are found in up to $54 \%$ of patients with chronic kidney disease (CKD) and congestive heart failure [4, 8-12]. The occurrence of depressive symptoms frequently begins with the onset of dialysis or even earlier in CKD of greater severity [13-15].

Although the problem of pain in ESRD patients has been recognized for more than 25 years, only a few studies, mainly focused on QoL, have investigated this subject. Pain is a common problem that has been both under-recognized and

263 hemiodialysis patients at the participating dialysis units

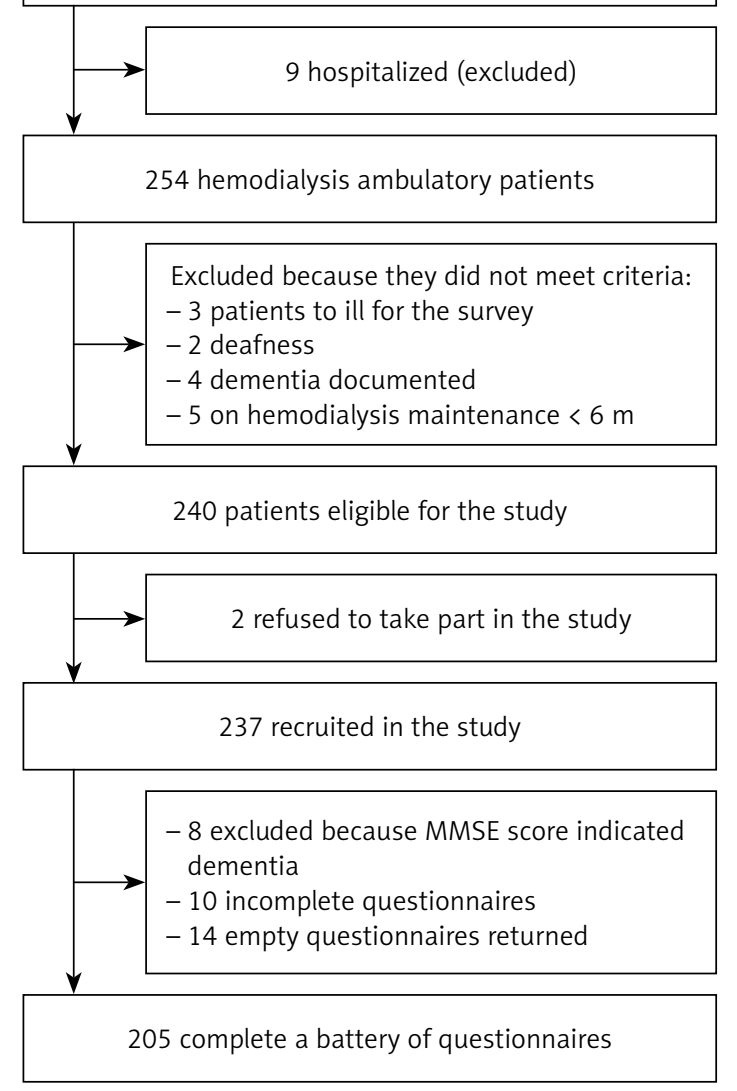

Figure 1. Study flow chart under-treated. Pain may be caused by underlying systemic diseases such as diabetic neuropathy or polycystic kidney disease, a comorbidity such as peripheral vascular disease or be due to routine aspects of renal replacement therapy (from needle insertions, or from muscle cramps during the procedure) and complications arising from dialysis access sites. Pain may result from calciphylaxis and renal osteodystrophy, which are unique to ESRD, or may develop during the patient's lifetime on dialysis. As in the general population, musculoskeletal pain is the most common symptom of chronic pain syndromes.

Some investigators have reported a higher prevalence of pain (51\%-73\%) among patients undergoing maintenance hemodialysis (Campbell et al., unpublished data, 2006; Haggiag et al., unpublished data, 2006), while others have reported a lower prevalence of pain (about 30-37\%) in these patients [16]. Chronic pain is a known factor affecting QoL, and intense, prolonged, self-reported pain may mask or induce depression/anxiety symptoms in some patients [17]. Hence, the next question is: to what extent do chronic pain and/ or depression influence the long-term outcome in ESRD patients?

In this study, we assessed the prognostic significance of chronic pain, depression/anxiety and QoL on 6-year survival in a cohort of clinically stable hemodialysis patients.

\section{Material and methods}

\section{Design and patients}

Initially, 240 ambulatory dialysis patients who met the inclusion criteria were asked to complete questionnaires concerning depression/anxiety symptoms, mental functioning, pain and quality of life in the period from February 2006 to May 2006. Patients were recruited from three dialysis centers in Lower Silesia, Poland (University Hospital $n=82$; Municipal Hospital (public) $n=49$; International Dialysis Center (private) $n=97$ ); the recruitment process is shown in Figure 1 . All patients received hemodialysis free of charge (i.e. paid for by the National Health Fund).

Patients meeting the following criteria were eligible for inclusion: (i) > 6 months on dialysis, (ii) standard hemodialysis (low-flux) three times a week, (iii) not hospitalized at the time of assessment, (iv) no physical impairments that would prevent the completion of the questionnaires. If blindness or an inability to write due to skeletal deformities was the only physical impairment, assistance in completing the questionnaires was provided. Exclusion criteria were: (i) dementia based on medical records or as assessed by the Mini-Mental State Examination (MMSE) that 
was provided for all patients 65 years and above, (ii) addiction to alcohol/psychoactive drugs, (iii) antidepressive treatment.

In the MMSE assessment, the cut-off for dementia in patients 65 years and older with higher education was $\geq 27$ points, while for those with a low education level it was $\geq 23$ points.

The study was approved by the Bioethics Committee of the Wroclaw Medical University.

\section{Clinical parameters}

In addition to demographic factors, baseline clinical data were recorded from electronic/written medical records and included cause of ESRD, maintenance dialysis, presence of residual diuresis, blood pressure, hemoglobin level, serum albumin, calcium-phosphorus product, parathyroid hormone (PTH), C-reactive protein (CRP) and parameters reflecting dialysis dose and adequacy. The aforementioned routine laboratory tests were performed at the hospitals' central analytical laboratory as part of standard patient care.

\section{Methods}

Participants completed a battery of previously validated questionnaires in the Polish language as well as a demographic appendix and anamnesis pain sheet. The Hospital Anxiety and Depression Scale (HADS:A and HADS:D), the 36-item Short Form Health Survey Questionnaire (SF-36) and the MMSE (for those 65 years or older) were provided. Two pain measurement tools (Verbal Rating Scale (VRS) and Visual Analog Scale (VAS)) were used to identify patients with chronic pain.

\section{Pain assessment}

Patients were asked to record their current level of pain (as assessed on a four-point categorical VRS and on a VAS consisting of a horizontal 100$\mathrm{mm}$ line) on a questionnaire $[18,19]$. The VRS in this study consisted of a series of adjectives reflecting varying degrees of pain severity ranging from "no pain" to "the most extreme pain". Patients circled the adjective that best described how severe their pain was. Numbers were provided along with adjectives - none (0), mild (1-3), moderate (4-6), severe (7-10) - to enhance interpretation of the scale. Additionally, a question about the first appearance of pain over time was incorporated. Chronic pain was defined as pain intensity reaching $\geq 1$ point(s) in both scales (more than $10 \mathrm{~mm}$ in the VAS and with at least "mild" being circled in the VRS) lasting more than 3 months. When less than $10 \mathrm{~mm}$ was marked in the VAS or "none" circled in the VRS, the patient was transferred to the group without chronic pain.

\section{Depression and Anxiety Symptoms}

The HADS [20] was developed as a tool for the identification of anxiety disorders and depression in patients in non-psychiatric hospital clinics. It consists of 14 items equally divided between anxiety and depression subscales. Each item is rated on a scale of 0 to 3 . Responders choose the response that most accurately describes how they have been feeling during the past. For both anxiety and depression scores, $0-7$ is considered normal, 8-10 mild and > 10 severe anxiety or depression. A review of several studies has shown that HADS has good psychometric properties (e.g. internal reliability and test-retest reliability) and is also capable of effectively assessing anxiety and depressive disorders in various health settings and in the general population $[11,21]$.

All included patients were treated according to the intention-to-treat principle. Study patients who scored $\geq 8$ in the HADS:A (the anxiety subscore of the HADS) as well as in HADS:D (the depression subscore of the HADS) were referred to a psychiatrist for confirmation of depression/anxiety disorders and treatment.

\section{Quality of life}

The Medical Outcome Study (MOS) 36-item Short Questionnaire Health Survey (SF-36 or RAND 36) was administered to evaluate general quality of life. This assessment tool consists of the following generic items: general health perception $(\mathrm{GH})$, physical functioning (PF), role limitations caused by physical health problems (RP), bodily pain (BP), vitality or energy (VT), mental health or emotional well-being $(\mathrm{MH})$, role limitations caused by emotional health problems (RE) and social functioning (SF) [22]. SF-36 has been translated and validated in many countries and for different chronic illnesses including ESRD [23, 24].

\section{Follow-up and survival analysis}

Patients were followed for 6 years after enrollment. Survival time was calculated as the number of months from the baseline assessment until death.

In the statistical analysis, kidney transplantation, transfer out of the facility and change of dialysis modality were recorded as censored observations.

The primary end-point was all-cause mortality. Causes of death were defined according to the European Renal Association-European Dialysis and Transplant Association (ERA-EDTA) coding system [25].

\section{Statistical analysis}

Differences between groups were analyzed using ANOVA or the Mann-Whitney $U$ test for con- 
tinuous variables and the $\chi^{2}$ test for categorical variables. A two sided $p$-value less than 0.05 was considered statistically significant.

Kaplan-Meier analysis was used to estimate survival from the time of pain/depression/QoL assessment to 6 years after evaluation. Univariate analysis was performed using log-rank tests to compare Kaplan-Meier survival curves. Cox proportional hazards regression was used to predict survival. The day of study recruitment was taken as the starting point for the analysis of patient survival (prevalent patient survival).

\section{Results}

Two hundred fifty-four prevalent ambulatory dialysis patients were considered for screening, of whom 205 were finally enrolled in the study (see study flow chart in Figure 1).

The response/consent rate was 205/240 (85.4\%). Eight patients older than 65 years were not eligible because of a high MMSE score (indicating cognitive deficits), ten questionnaires were incomplete and 14 patients did not respond. Finally, 205 patients (41.5\% female) with an average age of $60.3 \pm 13.8$ years (age range: $19-87$ years) were enrolled in the study as well as for survival analysis. A summary of demographic and clinical data is shown in Table I. Other clinical and biochemical measures for the entire cohort are displayed in Tables II and III.

\section{Chronic pain}

One hundred thirty (63.4\%) maintenance hemodialysis patients suffered from chronic pain of any cause as reported in the two tools of pain measurement ( $\geq 10 \mathrm{~mm}$ in the VAS and at least "mild" pain in the VRS).

Based on patients' responses in the anamnesis pain questionnaire, $84 \%$ of patients with pain (108) had bone-joint-muscle pain. The locations of pain were as follows: head $26 \%$ (35), neck and shoulders $20.6 \%$ (27), back $13.7 \%$ (18), lumbar region $25 \%$ (33), chest $10 \%$ (13), bones in general $6.8 \%$ (9), lower extremity $28 \%$ (7), knee $15 \%$

Table I. Baseline demographics and clinical characteristics $(n=205)$

\begin{tabular}{|c|c|c|}
\hline Parameter & $N$ or mean \pm SD & Percentage or median \\
\hline Gender, female/male & $85 / 120$ & $41.5(58.5 \%)$ \\
\hline Age & $60.3 \pm 13.8$ & Median 60 (range: 19-87) \\
\hline Maintenance hemodialysis [months] & $50.9 \pm 58$ & Median 26 (range: 7-300) \\
\hline Residual diuresis (> $300 \mathrm{ml} /$ day) & 79 & $38.5 \%$ \\
\hline Cause of ESRD: & $(n)$ & (\%) \\
\hline Glomerulonephritis & 55 & 26.8 \\
\hline Diabetic nephropathy & 46 & 22.4 \\
\hline Hypertensive nephropathy & 45 & 22 \\
\hline Polycystic kidney disease & 22 & 10.7 \\
\hline Pyelonephritis & 16 & 7.9 \\
\hline Other/unknown & 21 & 10.2 \\
\hline Comorbidities: & $(n)$ & $(\%)$ \\
\hline Hypertension & 119 & 58 \\
\hline Cardiovascular disease $\mathrm{e}^{\mathrm{a}}$ & 134 & 65 \\
\hline Diabetes mellitus & 48 & 23.4 \\
\hline Vascular access: & $(n)$ & $(\%)$ \\
\hline Native AVF single needle & 17 & 8.3 \\
\hline Native AVF two needles & 157 & 76.6 \\
\hline Temporal catheter & 7 & 3.4 \\
\hline Permanent catheter & 22 & 10.7 \\
\hline PTFE prosthesis & 2 & 1 \\
\hline
\end{tabular}

${ }^{a}$ Cardiovascular disease: stroke, myocardial infarction/revascularization, heart failure, coronary heart disease, peripheral occlusive artery disease, aortic aneurysm. AVF-arteriovenous fistula, ESRD - end-stage renal disease, PTFE - polytetrafluoroethylene. 
Table II. Baseline characteristics in the cohort, subgroups with pain ( $\geq 1$ VRS and VAS) and without pain

\begin{tabular}{|c|c|c|c|c|c|c|c|}
\hline \multirow[t]{2}{*}{ Parameter } & \multicolumn{2}{|c|}{ Cohort $(n=205)$} & \multicolumn{2}{|c|}{ No pain $(n=75)$} & \multicolumn{2}{|c|}{$\begin{array}{c}\text { Chronic pain } \\
(n=130)\end{array}$} & \multirow{2}{*}{$\begin{array}{c}\text { Pain vs. no } \\
\text { pain (ANOVA) } \\
P \text {-value }\end{array}$} \\
\hline & Mean & SD & Mean & SD & Mean & SD & \\
\hline Maintenance HD [months] & 50.9 & 58 & 36.1 & 43.3 & 64.9 & 67.8 & 0.001 \\
\hline Age [years] & 60.3 & 13.8 & 62.7 & 14.1 & 58.9 & 13.6 & 0.060 \\
\hline 24-h dieresis [l] & 0.5 & 0.5 & 0.5 & 0.5 & 0.3 & 0.5 & 0.007 \\
\hline $\mathrm{BMI}\left[\mathrm{kg} / \mathrm{m}^{2}\right]$ & 25.2 & 4.2 & 25.0 & 4.1 & 25.4 & 4.2 & 0.485 \\
\hline SBP before HD [mm Hg] & 134.7 & 25.4 & 136.4 & 26.5 & 133.8 & 24.7 & 0.479 \\
\hline DBP before $\mathrm{HD}[\mathrm{mm} \mathrm{Hg}]$ & 76.6 & 11.1 & 77.7 & 11.0 & 76.0 & 11.2 & 0.301 \\
\hline SBP after HD [mm Hg] & 128.2 & 26.2 & 131.3 & 25.7 & 126.6 & 26.4 & 0.217 \\
\hline DBP after HD [mm Hg] & 74.3 & 12.2 & 75.5 & 11.9 & 73.8 & 12.3 & 0.337 \\
\hline Albumin [g/dl] & 3.7 & 0.5 & 3.6 & 0.4 & 3.7 & 0.5 & 0.153 \\
\hline Hemoglobin [g/dl] & 10.9 & 2.5 & 10.6 & 1.6 & 11.1 & 2.8 & 0.214 \\
\hline CaxP & 51.0 & 17.4 & 47.2 & 17.1 & 53.1 & 17.3 & 0.019 \\
\hline PTH $[\mathrm{pg} / \mathrm{ml}]$ & 375.0 & 501.3 & 215.6 & 181.6 & 467.0 & 595.9 & $<0.001$ \\
\hline URR & 0.62 & 0.10 & 0.6 & 0.1 & 0.6 & 0.1 & 0.748 \\
\hline $\mathrm{kt} / \mathrm{V}$ & 1.14 & 0.26 & 1.1 & 0.2 & 1.2 & 0.3 & 0.171 \\
\hline kt/V weekly & 3.49 & 0.59 & 3.4 & 0.6 & 3.5 & 0.6 & 0.154 \\
\hline Mean UF per session [ml] & 2671.7 & 848.7 & 2610.7 & 857.9 & 2706.9 & 844.7 & 0.435 \\
\hline $\mathrm{CRP}[\mathrm{mg} / \mathrm{l}]$ & 13.3 & 1.6 & 12.1 & 15.0 & 14.2 & 23.7 & 0.499 \\
\hline HADS:A & 5.7 & 3.6 & 4.3 & 3.4 & 6.5 & 3.4 & $<0.001$ \\
\hline HADS:D & 6.0 & 4.2 & 4.6 & 4.0 & 6.8 & 4.1 & $<0.001$ \\
\hline SF-36 GH & 42.3 & 19.0 & 49.1 & 19.6 & 38.4 & 17.7 & $<0.001$ \\
\hline SF-36 BP & 60.0 & 31.8 & 86.8 & 22.0 & 44.5 & 25.6 & $<0.001$ \\
\hline SF-36 PF & 42.5 & 31.4 & 52.6 & 31.7 & 36.6 & 29.8 & $<0.001$ \\
\hline SF-36 RP & 46.8 & 44.2 & 57.4 & 44.3 & 40.5 & 43.1 & 0.008 \\
\hline SF-36 RE & 63.1 & 44.7 & 70.8 & 42.6 & 58.5 & 45.4 & 0.062 \\
\hline SF-36 VT & 49.6 & 21.9 & 59.2 & 22.4 & 44.1 & 19.7 & $<0.001$ \\
\hline SF-36 MH & 63.1 & 20.7 & 69.9 & 19.2 & 59.2 & 20.6 & $<0.001$ \\
\hline SF-36 SF & 66.0 & 28.8 & 77.4 & 26.0 & 59.5 & 28.4 & $<0.001$ \\
\hline
\end{tabular}

$B M I$ - body mass index (calculated from post-dialysis body mass), SBP - systolic blood pressure, DBP - diastolic blood pressure, CaxP - calcium-phosphorus product, URR - urea reduction rate, UF - ultrafiltration, HADS - Hospital Anxiety and Depression Scale (HADS:A anxiety, HADS:D - depression), SF-36 (Short Form 36 items) categories: GH - general health, PF-physical functioning, RP - role-physical limitation, $R E$ - role-emotional limitation, $B P$ - bodily pain, VT - vitality, MH - mental health, SF-social functioning.

(20), foot $23 \%$ (30), hand/wrist $7.6 \%$ (10), upper extremity $9.1 \%(12)$, abdomen $6.1 \%(8)$, hip $10 \%$ (14), calf cramps 53\% (70). Some reported pain in more than one location (hence the sum of locations exceeds 100\%). Moreover, 28\% (37) of patients reported continuous pain with an exacerbation during the dialysis sessions. The average duration of chronic pain was $20 \pm 14$ months, but often pain had persisted for 7 months prior to enrollment (the mode value was 7). Forty-three 
Table III. Characteristics of patients with depressive symptoms

\begin{tabular}{|c|c|c|c|c|c|c|c|}
\hline \multirow[t]{2}{*}{ Parameter } & \multicolumn{2}{|c|}{$\begin{array}{l}\text { Diagnosis of depres- } \\
\text { sion by psychiatrist } \\
\qquad(n=13)\end{array}$} & \multicolumn{2}{|c|}{$\begin{array}{c}\text { HADS:D }<8 \\
(n=141)\end{array}$} & \multicolumn{2}{|c|}{$\begin{array}{c}\text { HADS:D } \geq 8 \\
(n=62)\end{array}$} & \multirow{2}{*}{$\begin{array}{c}\text { HADS:D }<8 \\
\text { vs. } \geq 8 \\
\text { (ANOVA) } \\
P \text {-value }\end{array}$} \\
\hline & Mean & SD & Mean & SD & Mean & SD & \\
\hline Maintenance HD [months] & 67.3 & 78.6 & 53.3 & 56.9 & 56.7 & 70.9 & 0.634 \\
\hline Age [years] & 49.8 & 16.8 & 55.0 & 16.1 & 57.6 & 15.0 & 0.302 \\
\hline 24-h dieresis $[\mathrm{l}]$ & 0.3 & 0.5 & 0.4 & 0.5 & 0.3 & 0.5 & 0.232 \\
\hline $\mathrm{BMI}\left[\mathrm{kg} / \mathrm{m}^{2}\right]$ & 26.2 & 2.5 & 25.0 & 4.3 & 25.7 & 3.9 & 0.177 \\
\hline SBP before HD [mm Hg] & 137.9 & 17.8 & 132.7 & 23.9 & 139.2 & 27.9 & 0.090 \\
\hline DBP before HD [mm Hg] & 77.5 & 7.5 & 75.7 & 10.8 & 78.5 & 11.7 & 0.068 \\
\hline SBP after HD [mm Hg] & 132.9 & 21.2 & 128.0 & 25.4 & 128.8 & 28.1 & 0.994 \\
\hline DBP after HD [mm Hg] & 74.2 & 7.9 & 74.5 & 12.0 & 74.1 & 12.5 & 0.895 \\
\hline Albumin $[\mathrm{g} / \mathrm{dl}]$ & 3.7 & 0.4 & 3.7 & 0.5 & 3.6 & 0.5 & 0.343 \\
\hline Hemoglobin [g/dl] & 11.0 & 1.4 & 11.1 & 2.7 & 10.5 & 1.8 & 0.142 \\
\hline CaxP & 46.4 & 13.1 & 51.0 & 17.4 & 51.8 & 18.6 & 0.933 \\
\hline PTH $[\mathrm{pg} / \mathrm{ml}]$ & 602.0 & 742.7 & 343.2 & 451.2 & 445.0 & 594.9 & 0.546 \\
\hline URR & 0.6 & 0.1 & 0.6 & 0.1 & 0.6 & 0.1 & 0.850 \\
\hline $\mathrm{kt} / \mathrm{V}$ & 1.2 & 0.4 & 1.1 & 0.3 & 2.7 & 12.5 & 0.727 \\
\hline kt/V weekly & 3.5 & 0.8 & 4.2 & 8.2 & 3.5 & 0.6 & 0.809 \\
\hline Mean UF per session [ml] & 3166.7 & 957.6 & 2658.2 & 846.3 & 2701.6 & 859.8 & 0.555 \\
\hline CRP $[\mathrm{mg} / \mathrm{l}]$ & 22.9 & 26.6 & 12.8 & 20.3 & 15.7 & 21.9 & 0.473 \\
\hline VRS & 2.9 & 2.3 & 1.8 & 2.3 & 3.3 & 4.6 & 0.001 \\
\hline HADS:A & 8.0 & 4.7 & 4.6 & 2.6 & 8.1 & 4.3 & 0.006 \\
\hline HADS:D & 9.1 & 3.1 & 3.7 & 2.2 & 11.1 & 2.9 & $<0.001$ \\
\hline HADS:A + D & 17.1 & 5.4 & 8.4 & 3.9 & 19.2 & 5.9 & $<0.001$ \\
\hline SF-36 GH & 38.3 & 21.2 & 46.1 & 19.2 & 34.3 & 16.0 & $<0.001$ \\
\hline SF-36 BP & 39.8 & 27.4 & 65.7 & 30.5 & 48.2 & 31.4 & 0.007 \\
\hline SF-36 PF & 37.1 & 26.7 & 49.6 & 30.9 & 27.1 & 26.8 & 0.109 \\
\hline SF-36 RP & 37.5 & 47.1 & 49.3 & 43.6 & 41.4 & 45.4 & 0.845 \\
\hline SF-36 RE & 50.0 & 48.2 & 71.7 & 40.5 & 44.6 & 47.9 & 0.699 \\
\hline SF-36 VT & 34.6 & 18.9 & 55.0 & 20.4 & 38.3 & 20.9 & 0.011 \\
\hline SF-36 MH & 42.3 & 18.4 & 68.8 & 18.5 & 50.9 & 20.1 & 0.010 \\
\hline SF-36 SF & 53.1 & 25.6 & 70.7 & 26.2 & 55.8 & 31.8 & 0.269 \\
\hline
\end{tabular}

$B M I$ - body mass index (calculated from post-dialysis body mass), SBP - systolic blood pressure, DBP - diastolic blood pressure, CaxP calcium-phosphorus product, URR - urea reduction rate, UF - ultrafiltration, VRS - mean intensity of pain, HADS - Hospital Anxiety and Depression Scale (HADS:A - anxiety, HADS:D - depression), SF-36 (Short Form 36 items) categories: GH - general health, PF - physical functioning, $R P$ - role-physical limitation, $R E$ - role-emotional limitation, $B P$ - bodily pain, VT - vitality, MH - mental health, SF - social functioning.

percent of patients reported pain intensity as being "mild" in the VRS with a mean VAS of 3.07 $\pm 1.2 \times 10 \mathrm{~mm}$, while $57 \%$ of responders indicated "moderate" pain in the VRS with a mean VAS of $5.01 \pm 1.3 \times 10 \mathrm{~mm}$.
Patients with chronic pain had been on maintenance dialysis for a longer period of time and demonstrated a lower residual renal function (diuresis). Moreover, patients reporting pain had higher levels of calcium-phosphorus product 


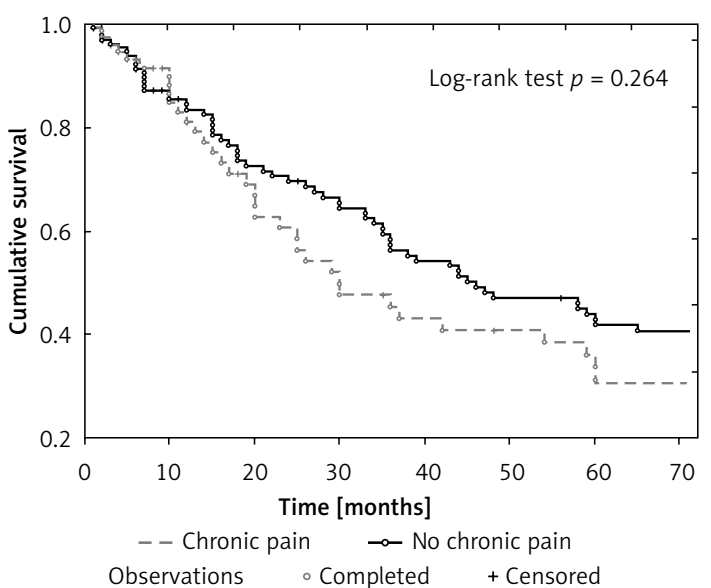

Figure 2. Comparison of survival in patients with $(n=130)$ and without $(n=75)$ chronic pain

$(\mathrm{Ca} \times \mathrm{P})$ and PTH concentrations than those without pain (Table II). Both groups showed comparable indices of dialysis adequacy. Significant differences (pain versus no pain) were found in the anxiety (HADS:A), depression (HADS:D) and QoL scales (Table II).

Analysis of 6-year survival in patients with any pain and in those without any reported pain did not show significant differences (Figure 2). Cox proportional hazards regression excluded pain as a factor influencing survival even when the subgroup of patients with moderate pain ( $\geq 4$ in the VRS) was extracted and separately analyzed (logrank test $p=0.573$ ).

\section{Anxiety and depression}

Sixty-two patients with scores $\geq 8$ points in the HADS were referred to a psychiatrist for confirmation of the diagnosis. Ten patients did not give consent for evaluation by a psychiatrist. Of the 52 evaluated patients, 13 (6\%) were diagnosed with depressive/anxiety disorders.

There were no significant differences in distributions of demographic and clinical parameters between patients with high $(\geq 8 ; n=62)$ or low $(<8 ; n=143)$ depression scores at baseline (Table III). The two groups differed in pain intensity (VRS $(p=0.001))$ and in 4/8 categories of QoL $(\mathrm{GH}$, $\mathrm{BP}, \mathrm{VT}, \mathrm{MH} ; p<0.01)$. Anxiety disorders were less prominent in the cohort than depressive disorders and showed no influence on cumulative survival.

Cox proportional hazards regression showed that depressive symptoms, scored in the HADS:D, were an important predictor of survival in univariate as well as multivariate analysis. A comparison of the cumulative survival of dialysis patients with high $(\geq 8)$ and low $(<8)$ depression scores in the HADS:D revealed significantly better survival in less depressed patients (Figure 3; log-rank test $p=0.035$ ).

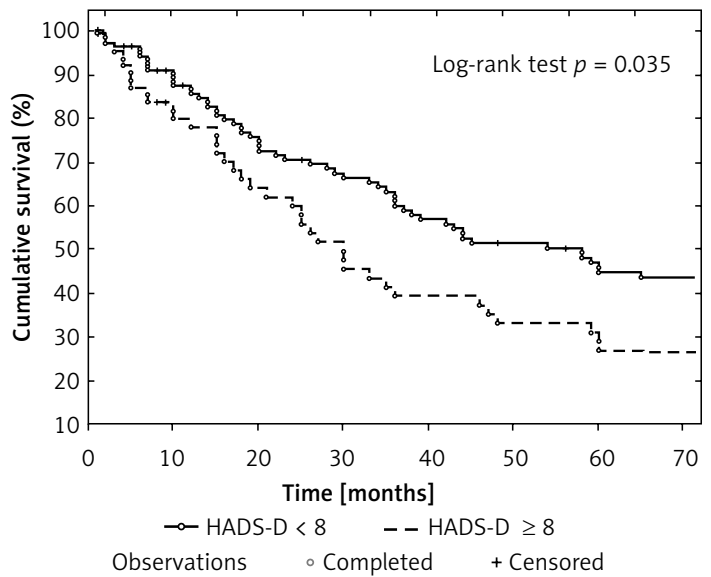

Figure 3. Comparison of survival in patients with high $(n=62)$ and low $(n=141)$ depression scores

An additional analysis of combined anxiety and depression symptoms (HADS:A + D) with a cut-off set at 13 points ( $<13$ vs. $\geq 13$ ) showed no difference in terms of survival (log-rank $p=0.121$ ) between the two groups.

\section{Correlations}

Negative correlations between pain (in the VRS) and QoL scores (higher VRS score = lower QoL) were observed in many categories: $\mathrm{GH}$ $(p=0.009 ; R=-0.12), \mathrm{BP}(p<0.001 ; R=-0.54), \mathrm{PF}$ $(p=0.05 ; R=-0.14), \operatorname{RP}(p=0.003 ; R=-0.21), \mathrm{RE}$ $(p=0.003 ; R=-0.22)$, VT $(p<0.001 ; R=-0.29)$, SF $(p<0.001 ; R=-0.43)$ and $\mathrm{MH}(p<0.001 ; R=-0.33)$. Comparable results indicating slight-to-moderate negative correlations were found between the pain VAS and QoL categories (data not shown).

Positive correlations were found between the depression scale (HADS:D) and pain (assessed using the VAS or the VRS) (Table IV). Many SF-36 categories displayed moderate negative correlations with the depression score (HADS:D). The strongest significant correlations $(R=-0.52)$ between QoL and depression scores were found for the mental (emotional) health and vitality categories (Table IV).

\section{Mortality rates}

During the follow-up period, 96 (46.8\%) patients died. The most common cause of death was cardiovascular disease, accounting for $45.8 \%$ (44) of deaths. According to the ERA-EDTA coding system (COD group) [25], the causes of death were as follows: myocardial ischemia and infarction (27), heart failure (9), cardiac arrest (8), infections (11), malignancies (13), cerebrovascular accident (4), miscellaneous (4) and unknown/unavailable (20).

\section{Survival analysis}

During 6 years of follow-up, 102 patients were censored at various time points due to renal trans- 
Table IV. Correlation coefficients for HADS:D, VAS VRS and SF-36

\begin{tabular}{|llc|}
\hline Relation of variables & $R$ & $P$-value \\
\hline VAS and HADS:D & 0.24 & $<0.001$ \\
\hline VRS and HADS:D & 0.27 & $<0.001$ \\
\hline SF-36-GH and HADS:D & -0.43 & $<0.001$ \\
\hline SF-36-BP and HADS:D & -0.42 & $<0.001$ \\
\hline SF-36-PF and HADS:D & -0.42 & $<0.001$ \\
\hline SF-36-RP and HADS:D & -0.28 & $<0.001$ \\
\hline SF-36-RE and HADS:D & -0.39 & $<0.001$ \\
\hline SF-36-V and HADS:D & -0.52 & $<0.001$ \\
\hline SF-36-SF and HADS:D & -0.33 & $<0.001$ \\
\hline SF-36-MH and HADS:D & -0.52 & $<0.001$ \\
\hline $\begin{array}{l}\text { VAS - current pain (visual analog scale), VRS - mean intensity } \\
\text { of pain, HADS:D - depression score, SF-36 (Short Form } 36 \text { items) } \\
\text { categories: GH - general health, PF - physical functioning, RP - } \\
\text { role-physical limitation, RE - role-emotional limitation, BP - bodily } \\
\text { pain, VT - vitality, MH - mental health, SF - social functioning. }\end{array}$
\end{tabular}

plantation (24), transfer out of the facility (75), recovery of renal function (1) and change of dialysis modality (switch to peritoneal dialysis; 2 ).

For the entire cohort, 1-, 2- 3-, 5- and 6-year patient survival on dialysis was $84 \%(\mathrm{SE}=0.03)$, $66 \%(\mathrm{SE}=0.04), 54 \%(\mathrm{SE}=0.04), 41 \%(\mathrm{SE}=0.04)$ and $37 \%(\mathrm{SE}=0.04)$, respectively.

Univariate analysis using log-rank tests to compare Kaplan-Meier survival curves or univariate Cox regressions (as appropriate) indicated that age $(p=0.008)$, serum albumin $(p<0.001)$, CRP level ( $p=0.004)$ and depression (as assessed using the HADS-D $(p=0.016)$ and HADS:A + D $(p=0.041))$ were significantly associated with survival. Other clinical and biochemical parameters, comorbidities (diabetes, cardiovascular burden) and pain as well as QoL outcome did not reach significance for patient survival.
The independent variables with a significant negative impact on 6-year survival according to the Cox proportional hazards regression model (Table V) were age $(p=0.041)$, lower albumin concentration ( $p=0.018)$ and more depressive symptoms reported in HADS-D $(p=0.016)$. Addition of the VRS (mean intensity of pain) to the abovementioned model diminished the impact of depression on patient survival. This may have resulted from a significant correlation between the VRS and the HADS-D (Table IV).

\section{Discussion}

Bodily pain and psychiatric distress are common symptoms in patients undergoing chronic hemodialysis. In the European population of dialysis patients, the relationship between chronic pain, depressive symptoms and survival has not been thoroughly investigated.

In this three-center, prospective cohort study, a high prevalence of chronic pain $(63.4 \%)$ was found. The most frequent complaints among our study subjects were of bone-joint-muscle pain (84\%). When patients have a high PTH level, it is usually a consequence of secondary hyperparathyroidism resistant to treatment. Pain localized in the hand (uni- or bilateral) was linked to arteriovenous fistula (ischemia or steal syndrome) and, in those with more than 5 years of maintenance hemodialysis, it was related to secondary amyloidosis. Dialysis-related amyloidosis that is due to the accumulation of $\beta_{2}$-microalbumin (resulting from poor clearance in low-flux membranes) typically manifests as pain in the shoulder or hand (carpal tunnel syndrome).

A group of Italian investigators found that 37 of 100 hemodialysis patients had chronic pain. In their analysis, the predominant cause of chronic pain (24 of 37) was osteoarthritis [16]. In a multicenter QoL study of 165 United States (U.S.) hemodialysis patients, Kimmel et al. found that $21 \%$

Table V. Cox proportional hazard regression model for 6-year survival time as a dependent variable

\begin{tabular}{|ccccc|}
\hline Model & Effect of & HR & $95 \% \mathrm{Cl}$ & $P$-value \\
\hline 1 & Age & 1.015 & $0.943-1.052$ & 0.041 \\
\cline { 2 - 5 } & Serum albumin & 0.581 & $0.305-1.274$ & 0.018 \\
\hline CRP & 0.997 & $0.973-1.015$ & 0.318 \\
\hline HADS:D & 1.055 & $0.944-1.121$ & 0.016 \\
\cline { 2 - 5 } & Sge & 0.968 & $0.953-1.00$ & 0.001 \\
\hline & CRP & 0.664 & $0.368-1.201$ & 0.428 \\
\hline & HADS:D & 0.997 & $0.973-1.015$ & 0.341 \\
\hline
\end{tabular}

VRS - verbal response scale (i.e. mean intensity of pain), HADS:D - depression score, CRP - C-reactive protein. 
of the patients reported pain [22]; however, the nature of the pain was not evaluated.

In our study, patients with pain (even mild) showed worse self-reported QoL (i.e. lower scores) than patients without pain. A negative impact of pain on QoL has been reported previously [22, 26]. An association was also found between pain and depressive symptoms. On performing subgroup analyses, we found that $18.3 \%$ of the cohort with chronic pain of greater intensity ( $>4$ in VRS) also had depressive symptoms (HADS:D > 8). The occurrence of intense chronic pain not only diminishes QoL but may induce depression [5, 22, 26].

Finally, Cox regression analysis showed that pain of any intensity did not affect the 6-year survival of hemodialysis patients.

The main finding in this study is that depression is a key predictor of mortality in the cohort of hemodialysis patients. Higher scores in the HADS:D were independently associated with poorer survival (Figure 3). The impact on 6-year survival was equally as important as the impact of traditional well-known factors such as age or serum albumin (Table V). Riezebos et al. also analyzed the influence of depressive symptoms (using the HADS-D with a cutoff $>7$ ) on the survival of Dutch dialysis patients. After adjustment for a series of clinical parameters, the association between depressive symptoms and mortality became even stronger [11].

A high level of depressive symptoms was found among hemodialysis patients as assessed in the HADS questionnaire (62 patients had scores of 8 or more points). Of the 52 patients interviewed by an experienced psychiatrist, 13 (25\%) were diagnosed with anxiety-depressive disorder. This accounts for $6 \%$ of the entire dialysis cohort, with possible under-scoring because 10 patients at high risk refused an interview diagnosis. Loosman et al. demonstrated that the HADS, a self-report rating scale, is a valid screening tool for detecting depression in ESRD patients [10]. HADS performs equally as well as the Beck Depression Inventory (BDI), which is also a self-report rating scale that includes somatic items.

Although a few studies involving a small number of patients showed no relationship between baseline depression scores and outcome [4, 27], most studies (using various self-reporting tools) underscored the impact of depression on mortality $[14,28-31]$.

There is no commonly accepted model that describes how depression increases the risk of morbidity (mainly cardiac) and mortality. Some authors report evidence of a link between depressive symptoms and major adverse cardiac events $[8,32]$. The increased mortality in patients with depressive symptoms reported in a Dutch study was mainly due to a high incidence of infec- tion-related deaths. This suggests a relationship between depression and inflammation or immune dysfunction. The relationship between depression and inflammation appears to be bidirectional [33]. Depression can result in the upregulation of inflammatory mediators as found in $50 \%$ of patients who receive interferon- $\alpha$ treatment [34]. In those patients, decreased brain concentrations of serotonin and dopamine resulted in the development of depression. The relationship between the altered serotonin levels seen in depressed patients and increased platelet aggregation and vasoconstriction (which can lead to coronary events) deserves mentioning. There are reports suggesting that depression is associated with changes in platelet function, and that selective serotonin reuptake inhibitors (SSRIs) may have antiplatelet activities [35, 36].

In another recent study on a relatively small group of hemodialysis patients, depression was not found to have any significant effect on the level of proinflammatory cytokines such as interleukin (IL)-1, IL-6 and tumor necrosis factor- $\alpha$ (TNF- $\alpha$ ). Moreover, no significant improvements in cytokine levels were observed after the administration of antidepressant therapy [37].

Depression is associated with activation of the hypothalamic-pituitary-adrenal axis [38]. There is growing evidence regarding the role of cytokines in interrupting the negative feedback mechanism of the hormonal axis via cortisol, leading to immune system stimulation $[39,40]$.

Another important issue associated with depressive symptoms and lower survival is nonadherence to the medical regimen. In hemodialysis patients, nonadherence can take a variety of forms including regular fluid overload or noncompliance with dietary sodium restriction. These findings demonstrate a negative correlation between level of medication adherence and depressive symptoms. Patients with depressive symptoms report a greater feeling of hopelessness, compromising cognitive abilities. Hopelessness, cognitive distortions and fatigue produce negative expectations of the future and lead to inadequate fluid and dietary adherence behaviors [41]. Akman et al. found a doubled likelihood of dietary nonadherence in depressed CKD patients when compared to patients without depression [42]. The strong association between depressive symptoms and withdrawal from dialysis therapy noted in the Dialysis Outcomes and Practice Patterns Study (DOPPS) is particularly noteworthy [9].

On analyzing mortality in dialysis patients, we noticed a very high frequency (45.8\%) of cardiovascular-related deaths. This has been reported in many ESRD registries worldwide [1]. Still, there remains a huge difference when mortality of dial- 
ysis patients is compared with that of the general population. For example, cardiovascular mortality rates in U.S. dialysis patients were found to be amplified by a factor of 5 to 500 (depending on the age group) when compared with that of the general population [43].

In the entire cohort 2-, 5- and 6-year survival in hemodialyzed patients was found to be $66 \%, 41 \%$ and $37 \%$, respectively. This outcome is comparable to data published by the European registry (ERA-EDTA), although greater caution should be exercised when interpreting survival in prevalent dialysis patients as opposed to incident dialysis patients (the starting point is the date of dialysis onset). Nevertheless, according to a recent European registry for a hemodialysis cohort (20022006), 2 - and 5 -year patient survival was $68 \%$ and $36 \%$, respectively [44].

One limitation of our study is the analysis of survival in prevalent dialysis patients (not incident dialysis patients). A potential bias is the unknown duration of depression in these patients prior to assessment.

In conclusion, our study underscores the fact that depression is an important psychosocial factor affecting not only QoL but also survival in hemodialysis patients. Chronic pain, although frequently observed among maintenance hemodialysis patients and often concomitant with depression, did not significantly lower the survival rate of these patients. The diagnosis and treatment of depression is a real challenge in hemodialysis patients; therefore, tools for screening depression/ anxiety should be routinely used in these patients. It remains to be determined how pharmacological and nonpharmacological approaches to treating depressive symptoms will impact on morbidity and mortality in hemodialysis patients.

\section{Acknowledgments}

We would like to thank the staff of the dialysis units for providing data on mortality during follow-up.

\section{Conflict of interest}

The authors declare no conflict of interest.

\section{References}

1. Canaud B, Tong L, Tentori F, et al. Clinical practices and outcomes in elderly hemodialysis patients: results from the Dialysis Outcomes and Practice Patterns Study (DOPPS). Clin J Am Soc Nephrol 2011; 6: 1651-62.

2. Cooper B, Penne E, Bartlett L, et al. Protein malnutrition and hypoalbuminemia as predictors of vascular events and mortality in ESRD. Am J Kidney Dis 2004; 43: 61-6.

3. Kakiya R, Shoji T, Tsujimoto Y, et al. Body fat mass and lean mass as predictors of survival in hemodialysis patients. Kidney Int 2006; 70: 549-56.
4. Kimmel PL, Peterson RA, Weihs KL, et al. Multiple measurements of depression predict mortality in a longitudinal study of chronic hemodialysis outpatients. Kidney Int 2000; 57: 2093-8.

5. Takaki J, Mutsuhiro N, Yano E. The relationship of quality of life and depression to mortality in hemodialysis patients. Dial Transpl 2005; 34: 568-73.

6. Kalantar-Zadeh K, Kopple JD, Block G, et al. Association among SF36 quality of life measures and nutrition, hospitalization, and mortality in hemodialysis. J Am Soc Nephrol 2001; 12: 2797-806.

7. Kimmel PL, Patel SS. Quality of life in patients with chronic kidney disease: focus on end-stage renal disease treated with hemodialysis. Semin Nephrol 2006; 26: 68-79.

8. Hedayati SS, Jiang W, O'Connor CM, et al. The association between depression and chronic kidney disease and mortality among patients hospitalized with congestive heart failure. Am J Kidney Dis 2004; 44: 207-15.

9. Lopes AA, Albert JM, Young EW, et al. Screening for depression in hemodialysis patients: associations with diagnosis, treatment, and outcomes in the DOPPS. Kidney Int 2004; 66: 2047-53.

10. Loosman WL, Siegert CE, Korzec A, et al. Validity of the hospital anxiety and depression scale and the Beck depression inventory for use in end-stage renal disease patients. Br J Clin Psychol 2010; 49: 507-16.

11. Riezebos RK, Nauta KJ, Honig A, et al. The association of depressive symptoms with survival in a Dutch cohort of patients with end-stage renal disease. Nephrol Dial Transplant 2010; 25: 231-6.

12. Son YJ, Choi KS, Park YR, et al. Depression, symptoms and the quality of life in patients on hemodialysis for end-stage renal disease. Am J Nephrol 2009; 29: 36-42.

13. Fischer MJ, Xie D, Jordan N, et al. CRIC Study Group Investigators. Factors associated with depressive symptoms and use of antidepressant medications among participants in the Chronic Renal Insufficiency Cohort (CRIC) and Hispanic-CRIC Studies. Am J Kidney Dis 2012; 60: 27-38.

14. Hedayati SS, Grambow SC, Szczech LA, et al. Physician-diagnosed depression as a correlate of hospitalizations in patients receiving long-term hemodialysis. Am J Kidney Dis 2005; 46: 642-9.

15. Tsai YC, Chiu YW, Hung CC, et al. Association of symptoms of depression with progression of CKD. Am J Kidney Dis 2012; 60: 54-61.

16. Fortina F, Agllata S, Ragazzoni E, et al. Chronic pain during dialysis. Pharmacologic therapy and its costs. Minerva Urol Nefrol 1999; 51: 85-7.

17. Davison SN. Chronic kidney disease: psychosocial impact of chronic pain. Geriatrics 2007; 62: 17-23.

18. Collins S, Moore R, McQuay H. The visual analogue pain intensity scale: what is moderate pain in millimeters? Pain 1997; 72: 95-7.

19. Serlin RC, Mendoza TR, Nakamura Y, et al. When is cancer pain mild, moderate or severe? Grading pain severity by its interference with function. Pain 1995; 61: 277-84.

20. Zigmond A, Snaith R. The Hospital Anxiety and Depression Scale. Acta Psychiatr Scand 1983; 67: 361-70.

21. Martin C, Tweed A, Metcalfe M. A psychometric evaluation of the Hospital Anxiety and Depression Scale in patients diagnosed with end-stage renal disease. Br J Clin Psychol 2004; 43: 51-4.

22. Kimmel PL, Emont SL, Newmann JM, et al. ESRD patient quality of life: symptoms, spiritual beliefs, psychosocial factors, and ethnicity. Am J Kidney Dis 2003; 42: 713-22. 
23. Meyer KB, Espindle DM, DeGiacomo JM, et al. Monitoring dialysis patients health status. Am J Kidney Dis 1994; 24: 267-79.

24. Kusztal M, Nowak K, Magott-Procelewska M, et al. Evaluation of health-related quality of life in dialysis patients. Personal experience using questionnaire SF-36. Pol Merkur Lekarski 2003; 14: 113-7.

25. van Dijk PC, Jager KJ, de Charro F, et al. Renal replacement therapy in Europe: the results of a collaborative effort by the ERA-EDTA registry and six national or regional registries. Nephrol Dial Transplant 2001; 16: 1120-9.

26. Barakzoy A, Moss A. Efficacy of the World Health Organization Analgesic Ladder to treat pain in end-stage renal disease. J Am Soc Nephrol 2006; 17: 3198-203.

27. Christensen AJ, Wiebe JS, Smith TW, et al. Predictors of survival among hemodialysis patients: effects of perceived family support. Health Psychol 1994; 13: 521-5.

28. Boulware LE, Liu Y, Fink NE, et al. Temporal relation among depression symptoms, cardiovascular disease events, and mortality in end-stage renal disease: contribution of reverse causality. Clin J Am Soc Nephrol 2006; 1: 496-504.

29. Drayer RA, Piraino B, Reynolds CF $3^{\text {rd }}$, et al. Characteristics of depression in hemodialysis patients: symptoms, quality of life and mortality risk. Gen Hosp Psychiatry 2006; 28: 306-12.

30. Kimmel PL, Peterson RA, Weihs KL, et al. Psychosocial factors, behavioral compliance and survival in urban hemodialysis patients. Kidney Int 1998; 54: 245-54.

31. Soucie JM, McClellan WM. Early death in dialysis patients: risk factors and impact on incidence and mortality rates. J Am Soc Nephrol 1996; 10: 2169-75.

32. Katon W, Ciechanowski P. Impact of major depression on chronic medical illness. J Psychosom Res 2002; 53 859-63.

33. Raison C, Capuron L, Miller A. Cytokines sing the blues: inflammation and the pathogenesis of depression. Trends Immunol 2006; 27: 24-31.

34. Capuron L, Miller A. Cytokines and psychopathology: lessons from interferon-alpha. Biol Psychiatry 2004; 56: 819-24.

35. Serebruany VL, Glassman AH, Malinin Al, et al.; SADHART Study Group. Platelet/endothelial biomarkers in depressed patients treated with the selective serotonin reuptake inhibitor sertraline after acute coronary events. Circulation 2003; 108: 939-44.

36. Serebruany VL, Suckow RF, Cooper TB, et al. Sertraline Antidepressant Heart Attack Randomized Trial. Relation ship between release of platelet/endothelial biomarkers and plasma levels of sertraline and $\mathrm{N}$-desmethylsertraline in acute coronary syndrome patients receiving SSRI treatment for depression. Am J Psychiatry 2005; 162: 1165-70.

37. Cilan H, Oguzhan N, Unal A, et al. Relationship between depression and proinflammatory cytokine levels in hemodialysis patients. Ren Fail 2012; 34: 275-8

38. Musselman D, Evans D, Nemeroff C. The relationship of depression to cardiovascular disease. Arch Gen Psychiatry 1998; 55: 580-92.

39. Badiou S, Cristol JP, Jaussent I, et al. Fine-tuning of the prediction of mortality in hemodialysis patients by use of cytokine proteomic determination. Clin J Am Soc Nephrol 2008; 3: 423-30.

40. Kiecolt-Glaser JK, Glaser R. Depression and immune function: central pathways to morbidity and mortality. J Psychosom Res 2002; 53: 873-6.
41. Theofilou P. Noncompliance with medical regimen in haemodialysis treatment: a case study. Case Rep Nephrol 2011; 2011: 476038

42. Akman B, Uyar M, Afsar B. Adherence, depression and quality of life in patients on a renal transplantation waiting list. Transpl Int 2007; 5: 1-6.

43. Foley R, Parfrey P, Sarnak M. Clinical epidemiology of cardiovascular disease in chronic renal disease. Am J Kidney Dis 1998; 32: S112-9.

44. Kramer A, Stel V, Zoccali C, et al.; ERA-EDTA Registry. An update on renal replacement therapy in Europe: ERA-EDTA Registry data from 1997 to 2006. Nephrol Dial Transplant 2009; 24: 3557-66. 